\title{
Enzyklopädie zum gestalteten Raum im Spannungsfeld zwischen Stadt und Landschaft
}

\author{
Werner Bätzing \\ Archiv für integrative Alpenforschung, Geyerswörthstr. 12, 96047 Bamberg, Germany \\ Correspondence to: Werner Bätzing (werner.baetzing@web.de) \\ Published: 18 March 2016
}

Lampugnani, V. M., Domhardt, K. S., und Schützeichel, R.: Enzyklopädie zum gestalteten Raum im Spannungsfeld zwischen Stadt und Landschaft, gta Verlag, Zürich, 510 S., ISBN-13: 978-3-85676-325-1, EUR 98, 2014.

Diese umfang- und materialreiche Enzyklopädie ist eine außergewöhnliche und außerordentliche Publikation, zu der es nichts Vergleichbares gibt. Gegenstand ist der „gestaltete Raum“ der gesamten Erde, also eine Thematik, die auch für die Disziplin Geographie von großem Interesse ist. Diese Rezension thematisiert zuerst Konzeption und Anspruch dieser Enzyklopädie, wobei sich die Darstellung sehr stark an der programmatischen Einleitung orientiert, danach wird die Umsetzung und der Inhalt vorgestellt, soweit dies bei einem so umfangreichen Werk möglich ist, und zum Schluss wird diskutiert, ob und inwieweit der selbst gestellte Anspruch eingelöst und erfüllt wird.

\section{Konzeption und Anspruch}

Die Erde ist zwar sehr stark durch menschliche Gestaltung geprägt, aber die konkreten intentionalen menschlichen Handlungen sind stets in ein komplexes Gefüge verschiedenster Faktoren eingebettet. Diese Enzyklopädie stellt den Versuch dar, die Mechanismen der Gestaltung aufzuspüren und sie in punktuellen Auslotungen aufzuzeigen, zu beschreiben und zu erklären (S. 6).

Da der gestaltete Raum in seiner Totalität nicht darstellbar ist, wird er in ausgewählte physische Raumelemente zergliedert, die jeweils Gegenstand eines Beitrages sind; auf diese Weise werden entscheidende Merkmale der Raumgestaltung konkret abgebildet (S. 6). Trotz der Wahl der Bezeichnung „Enzyklopädie“ verfolgt dieses Werk keine enzyklopädische Breite oder Vollständigkeit, sondern es stehen exemplarische Analysen im Mittelpunkt - die Herausgeber sprechen von einer „überschaubaren Triangulatur“ bzw. an anderer Stelle von einem „Rahmen, der Wissen organisiert und systematisiert" (S. 11) -, die das Wesentliche erfassen und weitere Vertiefungen anregen sollen (S. 7).

Da die einzelnen Raumelemente nicht isoliert von anderen Raumelementen betrachtet werden können, muss die inhaltliche Darstellung darauf in allen Einzelbeiträgen immer wieder Bezug nehmen. Und Raum wird dann ,als mosaikartig zusammengesetzte, zwar fragmentarische, aber doch zusammenhängende Einheit erfahrbar, die unter dem Gesichtspunkt ihrer Gestaltung erhellt wird“ (S. 7). Aus diesem Grund soll die Zusammenstellung der einzelnen Beiträge dieses Bandes weit mehr als die Summe der Einzelteile sein, denn diese sollen sich - auch dank zahlreicher Querverweise und Abbildungen - zu einem ,synoptischen Bild des gestalteten Raumes“ zusammenfügen (S. 12). Deshalb konzentriert sich dieses Werk darauf, diese Einzelelemente zum Gegenstand der Darstellung zu machen, und Modelle, die räumliche Zusammenhänge oder Ordnungen beschreiben, finden sich hier nicht (S. 7).

Die Gestaltungen, um die es in dieser Enzyklopädie geht, sind immer Ergebnis eines bewussten oder intentionalen Handelns. Da dieses aber stets durch eine Vielzahl anderer Faktoren mitbeeinflusst wird, werden diese Faktoren in den einzelnen Beiträgen stets herausgearbeitet, um die konkrete Gestaltung angemessen verstehen zu können; dabei liegt der Schwerpunkt der Darstellung auf dem Akt des Gestaltens und weniger auf dem Ergebnis (S. 8). Und unter „Gestaltung“ wird allein die Veränderung der physischen Form eines Elements verstanden, weshalb alle Veränderungen ausgeklammert werden, die allein im Kopf und im Bewusstsein des Betrachters stattfinden (S. 9).

Üblicherweise gilt die Stadt als das am stärksten vom Menschen gestaltete Element der Erde, aber dieses Werk will zeigen, dass auch Landschaft stark vom Menschen gestaltet 
sein kann, und wie ähnliche gestalterische Eingriffe und Absichten in Stadt und Landschaft auf Grund verschiedenster Abstufungen und Intensitäten unterschiedliche Formen annehmen können (S. 10). Und da jeder gestalterische Eingriff Teil eines geschichtlichen Prozesses ist, verfolgt dieses Buch eine ,historische Perspektive“, bei der die beiden letzten Jahrhunderte eine besonders große Rolle spielen (S. 10).

Zielgruppe dieses Werkes sind in erster Linie die Vertreter der planenden Wissenschaftsdisziplinen, in zweiter Linie die Vertreter anderer „raumwirksamer Disziplinen“ (darunter wird explizit auch die Geographie genannt) und schließlich die interessierten Laien, denen ein breiter Zugang zum Verständnis von Gestaltungsprozessen und kulturräumlichen Zusammenhängen eröffnet werden soll (S. 13).

\section{Umsetzung und Inhalt}

Der skizzierte Anspruch der Enzyklopädie erfordert einen inhaltlichen und methodischen Neuansatz, der zugleich Disziplinen übergreifend konzipiert sein muss. Dies wurde ermöglicht durch ein entsprechendes Forschungsprojekt, das vom Schweizerischen Nationalfonds seit 2009 gefördert und vom einem wissenschaftlichen Beirat begleitet wurde. Initiatoren und Moderatoren waren Dozenten der Professur für Geschichte des Städtebaus am Institut für Geschichte und Theorie der Architektur (gta) der Eidgenössischen Technischen Hochschule Zürich, die auch die Funktion der Herausgeberschaft übernahmen. Um den Blick auf den gestalteten Raum von der Perspektive der Stadt auf die sie umgebende Landschaft zu öffnen, mussten zahlreiche andere Disziplinen einbezogen werden. Die dazu notwendige Konzeption und Methodik wurde mittels zahlreicher Arbeitstagungen und Workshops erarbeitet, und darüber hinaus waren weitere Wissenschaftler bei der Auswahl der zentralen Raumelemente und bei ihrer wechselseitigen Verknüpfung beratend tätig. Auf diese Weise entstand ein mehrstufiger Forschungsprozess, bei dem alle Texte mehrere Peer Review-Verfahren durchliefen. Bei der Ausweitung der Perspektive der Architektur und des Städtebaus auf die Landschaft spielte offenbar der Biologe Hansjörg Küster eine wichtige Rolle, der „mit seinem universalen Wissen“ dem Projekt „unverzichtbare Denkanstöße“"gab (S. 509).

Insgesamt sind an diesem Werk 33 Wissenschaftler als Autoren beteiligt. Sie stammen zum großen Teil aus Architektur/Städtebau (11 Autoren), aus Kunstgeschichte (8 Autoren) und Geschichte (5 Autoren), während weitere Disziplinen (darunter Geographie und Philosophie) nur am Rand vertreten sind.

Die Herausgeber bezeichnen die von ihnen entwickelte Erarbeitung der Enzyklopädie als „transdisziplinär“. Um Missverständnisse zu vermeiden, muss klargestellt werden, dass sie transdisziplinär im Sinne einer integrativen Interdisziplinarität verstehen (als das akademische Zusammenwirken mehrerer Disziplinen), während die von Jürgen Mit- telstraß geprägte Definition von Transdisziplinarität $(\mathrm{Zu}-$ sammenarbeit von Wissenschaftlern mit Vertretern der Praxis/Alltagswelt) hier nicht gemeint ist.

Die „Enzyklopädie zum gestalteten Raum“ besteht bei einem Umfang von gut 500 Seiten im Großformat $(19,2 \times 28,0 \mathrm{~cm})$ aus lediglich 38 Einzelartikeln, die dafür aber jeweils 12-14 Druckseiten umfassen (lediglich der Artikel „Stadt“ fällt mit 21 Seiten aus diesem Rahmen), so dass jeder Artikel sehr material- und inhaltsreich ausfällt; dies ist aber auch notwendig, um die verschiedensten Faktoren, die bei der Gestaltung einen Einfluss besitzen, die geschichtlichen Entwicklungen sowie den Prozess des Gestaltens angemessen darstellen zu können. Alle Beiträge sind aufwendig bebildert (1-3 Abbildungen pro Doppelseite) - es gab sogar eine eigene Bildredaktion -, wobei die Abbildungen - oft historische Bilder und Luftbilder sowie historische Pläne die Texte auf eine sehr aussagekräftige Weise erweitern.

Die 38 Einzelbeiträge widmen sich den als zentral angesehen Raumelementen. Diese bestehen aus konkret abgrenzbaren und relativ kleinen Flächen (Baublock, Brücke, Brunnen, Einkaufszentrum, Flughafen, Friedhof, Hof usw.), aus linienhaften Flächen (Autobahn, Bahnanlagen, Landstraße, Weg usw.), aus zusammengesetzten, größeren Flächen (Stadt) sowie aus großflächigen Elementen (Landschaft, Meer, Wald usw.).

Es gibt zwar keine gemeinsame Gliederung der Beiträge, aber sie weisen doch ein gemeinsames Herangehen auf: Am Beginn steht eine etymologische Begriffsklärung und eine Definition, dann folgt die Genese des jeweiligen Elements vom allerersten Beginn bis heute, die oft in „Phasen des Wandels" untergliedert wird (Schwerpunkt 19. und 20. Jahrhundert), dann werden die gestaltprägenden Kräfte analysiert, und am Schluss steht manchmal ein Hinweis auf aktuelle Probleme und Herausforderungen. Zusammen mit der wichtigen Bebilderung, mit Anmerkungen und Literaturverzeichnis entstehen so kleine, präzise Monographien der jeweiligen Raumelemente, die sehr inhaltsreich sind.

Erstaunlich ist, dass der räumliche Bezugsrahmen dieser Enzyklopädie nirgends explizit thematisiert wird: Dieses Werk konzentriert sich auf Mitteleuropa bzw. auf ,,die westliche Welt" (Beispiele aus der Schweiz, Deutschland und den USA sind besonders prominent vertreten) oder auf „Europa und andere hochindustrialisierte Länder" (S. 412), was den Interessen und Bedürfnissen der Zielgruppe der planenden Disziplinen entspricht.

Alle 38 Einzelbeiträge zeichnen sich durch eine sehr hohe fachliche Kompetenz und eine sehr große Materialfülle aus, wodurch wichtige Einsichten in den Prozess der Raumgestaltung und viele neue Erkenntnisse ermöglicht werden. Für den Rezensenten war es z.B. interessant, dass die Anfänge der Autobahnen nicht im Fernverkehr, sondern im urbanen Raum liegen, dass die Eisenbahnen auf 2700 Jahre alte „Spurbahnen“ zurückgehen, oder dass der Begriff Landstraße nicht vom Land, sondern von der Landesherrschaft kommt. Allein schon diese gut strukturierte Informations- und Materialfül- 
le, die an zahlreichen Beispiele tiefe Einblicke in den Prozess der Raumgestaltung ermöglicht, macht diese Enzyklopädie zu einem wichtigen Werk.

\section{Bewertung}

Erfüllt jetzt diese Enzyklopädie den selbst gestellten Anspruch, anhand exemplarischer Beispiele die Vielfalt des gestalteten Raumes zu präsentieren und zentrale Mechanismen seiner Gestaltung aufzuspüren?

Als erstes fällt auf, dass die Auswahl der einzelnen Raumelemente - von kleinen Flächen bis hin zu sehr großflächigen Strukturen - uneinheitlich ist, so dass eine Zusammenschau im Sinne einer Synthese nicht leicht möglich ist, zumal ordnende oder systematisierende Beiträge bewusst ausgeschlossen werden. Die Querverweise im Text und vor allem die vielen Abbildungen, die Bezüge zwischen den einzelnen Beiträgen herstellen sollen (S. 12), können diese Schwäche jedenfalls aus geographischer Sicht - nicht wirklich ausgleichen.

Weiterhin fällt auf, dass der Beitrag „Berg“ nicht das Raumelement Berg thematisiert, sondern die Alpen bzw. die Berggebiete in Europa insgesamt, dass der Beitrag „Feld“ nicht ein einzelnes Feld behandelt, sondern die gesamten Agrarflächen, dass der Beitrag „Meer“ zwischen Aussagen zum offenen Meer und den Küsten changiert, und dass die Beiträge „Landschaft“ und „Wald“ sowieso flächenhaft ausgerichtet sind. Obwohl die Enzyklopädie den Anspruch formuliert, einzelne Raumelemente zu thematisieren (was die Beiträge von Architekten, Städtebauern und Planern berücksichtigen), sprengen die Beiträge zum Thema Kulturlandschaft dieses Raumkonzept fast immer, wodurch eine spürbare Asymmetrie entsteht, die eine Synthese stark erschwert.
Die Ursache scheint dem Rezensenten darin zu liegen, dass es zwischen den von Architekten gebauten Raumstrukturen und den Raumstrukturen der Kulturlandschaft im Prozess der Gestaltung doch sehr große Unterschiede gibt, auch wenn beiden durchaus intentionale Handlungen zu Grunde liegen. Dieser Unterschied wird nirgends explizit angesprochen, was eine Synthese zusätzlich erschwert. Ein entsprechender Beitrag am Ende des Werkes hätte dies gut thematisieren können, zumal der sehr lange Beitrag „Stadt“ von Magnago Lampugnani bereits de facto eine Art Synthese für den Bereich Architektur/Städtebau darstellt.

Fazit: Im Bereich der Synthese besitzt diese „Enzyklopädie zum gestalteten Raum“ einige Schwächen, aber die Bedeutung dieses Pionierwerkes zur Raumgestaltung ist so groß, dass es in jede geographische Bibliothek gehört. 